\title{
Contemporary Surgery in Infective Endocarditis
}

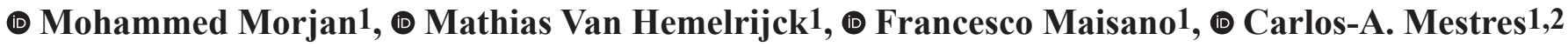

1Zurich University Hospital, Clinic of Cardiac Surgery, Zurich, Switzerland

2University of the Free State, Department of Cardiothoracic Surgery, Bloemfontein, South Africa

\begin{abstract}
Infective endocarditis is an uncommon disease but still carries high morbidity and mortality. The management of the patient with infective endocarditis changed over the last years with improvement of diagnostic tools and early aggressive medical and surgical treatment. The multidisciplinary approach is an accepted standard of practice and approximately $40-50 \%$ of patients discussed in endocarditis teams undergoes surgery. Timing of surgery
\end{abstract}

\section{Introduction}

Infective endocarditis (IE) is an uncommon disease, but with a significant related mortality and morbidity. Its incidence ranges between 3 to 10 per 100,000 per year and the same trends to rise $\mathrm{e}^{(1)}$.

Despite improvement in early diagnosis and surgical interventions with the introduction of a multidisciplinary approach for the management of patients with $\mathrm{IE}^{(2)}$, remains a debated issue, while complexity of surgery remains a characteristic of this pathology. Although isolated native valve endocarditis remains associated with acceptable morbidity and mortality, the same still high in the setting of multiple valve surgery and prosthetic infections.

Keywords: Infective endocarditis, endocarditis team, surgical treatment, epidemiology

morbidity and mortality has not substantially improved. There are multiple reasons behind such paradox including the new antibiotic resistance spectrum, the new risk patient profile and the introduction of new intracardiac devices associated with higher risk of endocarditis ${ }^{(3)}$. Such changes are directly associated with a new pattern of epidemiological features that should be interpreted as an important element affecting the contemporary therapeutic approach.

Address for Correspondence: Mohammed Morjan, Zurich University Hospital, Clinic of Cardiac Surgery, Zurich, Switzerland Phone: +41 432539497 e-mail: Mohammed.Morjan@usz.ch Received: 17.10.2019 Accepted: 11.11.2019

Cite this article as: Morjan M, Hemelrijck MV, Maisano F, Mestres CA. Contemporary Surgery in Infective Endocarditis. EJCM 2019;7(4):166-171.

DOI: $10.32596 /$ ejcm.galenos.2019.10.053

${ }^{\circ}$ Copyright 2019 by Heart and Health Foundation of Turkey (TÜSAV) / E Journal of Cardiovascular Medicine published by Galenos Publishing House. 


\section{Recent Epidemiological Changes}

Important changes occurred in the epidemiology of IE over the past couple of decades. While in previous times risk factors for endocarditis were pre-existing valvular abnormalities such as rheumatic disease and congenital heart defects, the widespread changes in health-care delivery in recent years have affected the clinical pattern of IE. Nowadays, the risk factors for IE include new intracardiac devices, more prosthetic valve implants, haemodialysis, drug use, immunosuppression and an increase in age and morbidity profile of the general population. Furthermore, changes in antibiotic therapy have influenced the spectrum of bacterial resistance worldwide.

Analysis of epidemiological data of large populations confirmed these changes. Slipczuk et al. performed a systematic review of the epidemiology of IE; hospitalbased (23,606 IE patients) and population-based (3,477 IE patients) studies were included ${ }^{(3)}$. In the large cohort of the hospital-based studies, the analysis showed significant changes in IE epidemiology. Patients were significantly older (1980s mean age $=45.3-2000 \mathrm{~s}$ mean age $=57.2$ years $)$, there were more male, the percentage of prosthetic valve IE increased (1960s 8.4\%-2000s 22.9\%), and significant increase in the frequency of staphylococcal infections (1960s 18.1\%-2000s 29.7\%) was noted; however, there was no significant difference with regards in-hospital mortality.

Pant et al. analysed the trends in the incidence of IE and the changes in the microbiological pattern from 2000 to 2011 in the United States; 457,052 IE patients were identified and an increase of the incidence of IE from $11 \%$ to $15 \%$ per 100,000 inhabitants was registered ${ }^{(4)}$. In addition, an increase in IE incidence was seen across all types of pathogens, especially in Staphylococcous IE (from 33\% in 2000 to $40 \%$ in 2011), Streptococcus (from $24.8 \%$ in 2000 to $27 \%$ in 2011), gram-negative (from $5.3 \%$ in 2000 to $8.2 \%$ in 2011 ) and fungal IE (from $0.6 \%$ in 2000 to $1.4 \%$ in 2011).

Bustamante-Munguira et al. analysed in an epidemiological study 34,399 IE patients registered between 1997 and 2014 in Spain ${ }^{(5)}$. They reported an increase in incidence of IE from $3.17 \%$ per 100,000 inhabitants in 1997 to $5.56 \%$ in 2014 with more prevalence in men; $15.7 \%$ of the cohort underwent surgical treatment over the whole period of time and the percentage of patients underwent surgery increased from $11.7 \%$ in 1997 to $17.8 \%$ in 2014. These patients presented with more organ dysfunction, especially renal failure. In this cohort, $84.3 \%$ received medical therapy and these patients were older and had more comorbidities. The mortality in the surgical patient was higher than in the non-operated patients but decreased over the time (32.7\% in 1997 to $22 \%$ in 2014); in addition, the mortality in the medical treated patients increased (14.9\% in 1997 to $21.1 \%$ in 2014). The mortality of patients undergoing surgery increased with age reaching $47.6 \%$ in those older than 85 years.

IE following surgical valve replacement or prosthetic valve endocarditis (PVE) is a very serious form of endocarditis; it represents $10-30 \%$ of all cases of $\mathrm{IE}^{(6)}$. In a recent publication, Østergaard et al. reported an incidence around 6/1000 per year among patients with a prosthetic heart valve $\mathrm{e}^{(7)}$. In such patients, the cumulative risk of IE was $2.8 \%$ and $4.5 \%$ at 5 and 10 years, respectively.

A new category of patients with prosthetic heart valves is those undergoing transcatheter aortic valve implantation (TAVI). In this category of patients PVE occurs with an incidence of $0.3 \%-1.2 \%$ per patient-year presenting comparable rates with PVE after surgical replacement ${ }^{(8,9)}$. However, a much higher incidence $(2.3 \%$ $3.4 \%$ ) per patient-year is reported in individual series or registries $^{(10,11)}$. The majority of these patients received a TAVI due to inoperability or high risk of conventional surgery; the treatment of such patients in case of PVE represents a medical and ethical challenge.

The epidemiology of IE has definitely changed over the last decades with its incidence trending upwards. This increase is multifactorial and probably related to the improvement in diagnostic tools, more use of medical devices, and an increase in patient age, with patients presenting with more comorbidities and an increase in 
staphylococcal infections. Such changes in epidemiology have an impact on the current management of patients with IE.

\section{The Endocarditis Team and the Importance of Surgery Timing}

As we stressed in the past, IE is a medical-surgical disease in which surgery is a part of the therapeutic process. The management of patients with IE in reference centres by a specialized team "Endocarditis team" is nowadays strongly recommended ${ }^{(12)}$. The functioning and organisation of an endocarditis team has been already described elsewhere ${ }^{(13)}$ and one of its most important functions is the selection of appropriate indication and timing of surgery. According to the European Society of Cardiology guidelines ${ }^{(12)}$, surgical timing is defined as emergent when surgery is performed within 24 hours, urgent when surgery is performed within a few days, or elective surgery when performed after 1 to 2 weeks of antibiotic therapy after surgical indication. In any case, we still miss a solid unequivocal definition of "early surgery".

The decision to perform surgery in IE remains a challenge because of the potential for acute and life-threatening complications, uncertain response to antibiotic therapy and pre-operative patient profile. As previously mentioned, around $40-50 \%$ of patients discussed in endocarditis team will need surgery. One of the most important issues discussed in the endocarditis team is "when to operate on?". The indications for surgery in the acute phase remain heart failure, newer conduction abnormalities, peri-annular complications and extravalvular spread and persistent sepsis despite aggressive and culture-oriented antibiotic therapy. However, the majority of such patients present with other systemic acute morbidity such as cerebral or systemic embolization. This must be considered when the surgical therapy is contemplated as those events could have an important impact on prognosis.

In particular, neurological events in the context of EI significantly influence the decision on the timing of the operation, as they can affect strongly post-operative morbidity and mortality. This is still a controversial matter generating considerable debate ${ }^{(14)}$. The decision on the surgical timing in these patients requires a balance between the urgency of the operation for cardiac indications versus the perceived risk of exacerbation of neurological injury. Our approach in this category of patients is individualized in most cases. However, in the absence of emergent indications to surgery, we prefer a wait-and-see approach of two to four weeks to reduce the risk of intracerebral haemorrhage, hypotension, or further embolization from cardiopulmonary bypass, and diffuse cerebral ischemia from altered vasoregulation.

Lalani et al. found that patients with IE receiving antibiotic treatment should undergo surgery within 4 weeks of admission, Kang et al. suggested what they called early intervention (within $48 \mathrm{~h}$ ) for patients with severe valvular regurgitation who have embolization and relevant vegetation dimensions $(>10 \mathrm{~cm})^{(15,16)}$. Early surgery within 48 hours of the acute event was supposed to be related to possible benefits in terms of mortality at the short- and long-term ${ }^{(16,17)}$. However, the Kang et al. study did not show mortality benefit at 90 days ${ }^{(16)}$. Lalani et al. confirmed a high 1-year mortality rate in patients with PVE but an advantage of early surgery in such group was not reported ${ }^{(18)}$.

The optimal surgical timing for patients with IE depends on a variety of factors such as clinical characteristics, compliance of the patient and presence of systemic acute and chronic comorbidity. An emergency operation could be indicated in some patients with acute heart failure or conduction abnormalities related to local aggressiveness of the disease and the pathogen. Although the indication for surgery should be considered in a multidisciplinary team discussion, the majority of cases should be individually addressed according to the pre-operative characteristics and risk profile.

\section{Contemporary Surgery for Endocarditis}

Surgery for IE remains challenging although surgical techniques have improved and surgeons with special 
dedication to this disease acquired skills and experience allowing them to face more and more complicated cases. Surgical therapy is a part of a complex multidisciplinary approach aiming to treat patients with IE. As stated, the profile of patients with IE changed, they are older with more comorbidities, and are admitted with systemic complications needing a meticulous evaluation and pretreatment. In addition, due to changes in antibiotic therapy and resistance spectrum, the nature of infection itself changed, it became more aggressive locally with frequent formation of abscesses and fistulas rending the surgical treatment more demanding. Timing and indication are crucial. At this point in time, there still exist not enough data indicating if early surgery is associated with benefit for short- and long-outcomes, however an individual approach, based on accurate analysis conducted by an experienced endocarditis team seem to be associated with more benefit.

Isolated native valve endocarditis confined to the leaflet tissue, theoretically represents the less challenging form of IE. Patients with isolated aortic valve IE could be electively operated due to valvulardysfunction after successful medical treatment. In the majority of these cases the mechanism of dysfunction is related to a perforation of one or more leaflet or the destruction of one component of the valvular apparatus. The indication for surgery in these cases follows the current guidelines for valve pathology ${ }^{(19)}$. Replacement of the native valve is traditionally performed to avoid recurrence of IE and avoid the long-term consequences of valvular dysfunction. Biological or mechanical prostheses could be implanted with good results. Toyoda et al. reported similar survival rate and incidence of re-operation at 12 years using both mechanical and biological prostheses in aortic and mitral position ${ }^{(20)}$. However, valve repair when feasible, particularly in mitral position, was associated with better long-term results ${ }^{(21)}$.

In case of urgent or emergent surgery, the procedure is usually more challenging. The indication could be related to acute valve dysfunction often with heart failure or local aggressiveness with abscess formation, de novo conduction disturbance with or without sepsis. The aortic valve is the mostly involved in these cases and the extension of the infection to the fibrous trigones requires extensive debridement with more demanding surgery including root replacement, double valve surgery and reconstruction of the destroyed anatomy ${ }^{(22)}$. Gillinov et al. reported on the surgical outcomes of 53 patients with native double IE over a 22-year period; no operative mortality was reported and the 10 -year actuarial survival was $73 \%^{(23)}$. Sheikh et al. reported outcomes of double valve surgery in IE in a cohort of 90 patients over a 26-year period, a significant inhospital mortality $(15.6 \%)$ was reported ${ }^{(24)}$. The long-term survival was $51 \%$ inclusive of patients treated for PVE.

PVE represents the most serious form of endocarditis. In the majority of cases, radical surgery is the only treatment able to modify the natural history of the disease. PVE surgery could be demanding and should be performed by a skilled and experienced team. The challenging nature of such surgery is related to a number of factors. As these are re-operations with patients frequently in suboptimal pre-operative condition, they are associated with higher morbidity and mortality in comparison with first-time surgery ${ }^{(25)}$. From the surgical standpoint, these procedures are frequently demanding, especially in the case of abscess formation. The need for extended surgical procedures like root replacement or re-replacement are cumbersome and entail long periods of cardiac ischemia. Commercially available composite grafts must be used and the role of homograft replacement has been well identified over the years, although availability is an issue. In spite of the demanding technical nature of such procedures, Musci et al. reported satisfactory early- and long-term results ${ }^{(26)}$.

In case of destruction of the fibrous skeleton of the heart, a more extended reconstruction may be required. The so-called "commando" or "hemi-commando" operations could be an option (Figure 1A-D). Elgharably et al. reported one-year survival of $91 \%$ and a 3 -year survival of $82 \%$ when the hemi-commando procedure was performed, with recent additional information on the reconstruction of the aortomitral fibrosa in general with different techniques ${ }^{(27-29)}$. Moreover, such operations 


Review Article 170 EJCM) of EJournal
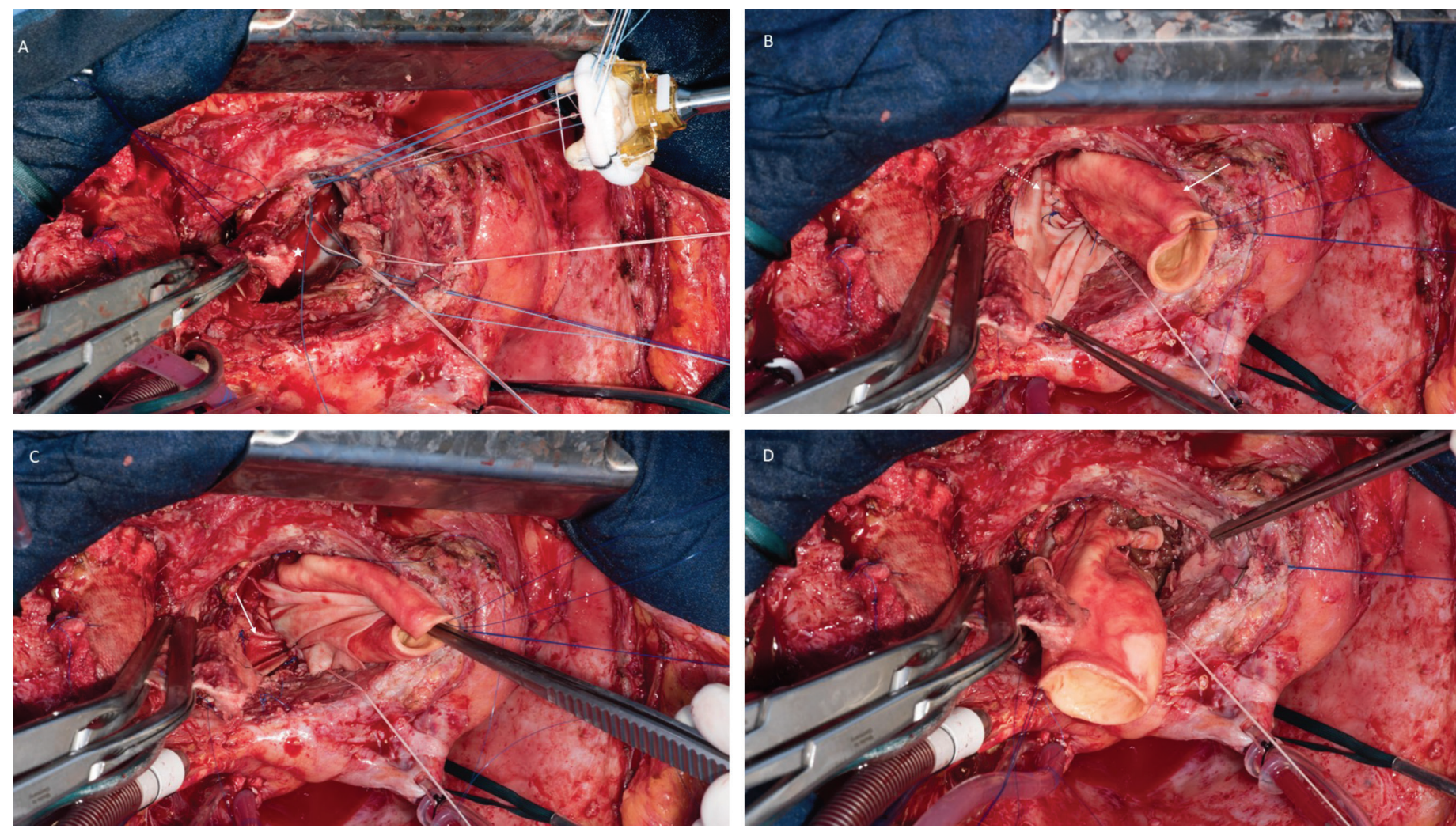

Figure 1A-D. The so-called commando operation

require long cardiopulmonary bypass, ischemic and overall operative times, which increase further the risk of postoperative complications. Extreme cases of cardiac destruction may require heart transplantation as it has been reported earlier. This is an old option in desperate cases but requires a microbiologically controlled status ${ }^{(30)}$.

\section{Conclusion}

IE is still a challenging disease requiring complex diagnostic and treatment efforts. The endocarditis team approach is the current standard of practice with clear functions aiming to provide the best indication, timing of surgery and overall care for the patient. Surgery of endocarditis became more and more demanding due to changing patterns of infection, more local aggressiveness and the pre-operative condition of patients.

\section{Ethics}

Peer-review: Internally and externally peer-reviewed.

\section{Authorship Contributions}

Surgical and Medical Practices: M.M., F.M., C.A.M., Concept: M.M., F.M., C.A.M., Design: M.M., M.V.H., F.M., C.A.M., Data Collection or Processing: M.M., M.V.H., C.A.M., Analysis or Interpretation: M.M., M.V.H., F.M., C.A.M., Literature Search: M.M., C.A.M., Writing: M.M., M.V.H., F.M., C.A.M.

Conflict of Interest: No conflict of interest was declared by the authors.

Financial Disclosure: The authors declared that this study received no financial support.

\section{References}

1. Erichsen P, Gislason GH, Bruun NE. The increasing incidence of infective endocarditis in Denmark, 1994-2011. Eur J Intern Med 2016;35:95-9.

2. Chambers J, Sandoe J, Ray S, et al. The infective endocarditis team: recommendations from an international working group. Heart 2014;100:524-7. 
3. Slipczuk L, Codolosa JN, Davila CD, et al. Infective endocarditis epidemiology over five decades: a systematic review. PLoS One 2013;8:e82665.

4. Pant S, Patel NJ, Deshmukh A, et al. Trends in infective endocarditis incidence, microbiology, and valve replacement in the United States from 2000 to 2011. J Am Coll Cardiol 2015;65:2070-6.

5. Bustamante-Munguira J, Mestres CA, Alvarez P, et al. Surgery for acute infective endocarditis: epidemiological data from a Spanish nationwide hospital-based registry. Interact Cardiovasc Thorac Surg 2018;27:498-504.

6. Glaser N, Jackson V, Holzmann MJ, Franco-Cereceda A, Sartipy U. Prosthetic Valve Endocarditis After Surgical Aortic Valve Replacement. Circulation 2017;136:329-31.

7. Østergaard L, Valeur N, Ihlemann N, et al. Incidence and factors associated with infective endocarditis in patients undergoing left-sided heart valve replacement. Eur Heart J 2018;39:2668-75.

8. Amat-Santos IJ, Messika-Zeitoun D, Eltchaninoff $\mathrm{H}$, et al. Infective endocarditis after transcatheter aortic valve implantation: results from a large multicenter registry. Circulation 2015;131:1566-74.

9. Regueiro A, Linke A, Latib A, Ihlemann N, Urena M, Walther T. Association Between Transcatheter Aortic Valve Replacement and Subsequent Infective Endocarditis and In-Hospital Death. JAMA 2016;316:1083-92.

10. Yoon SH, Bleiziffer S, De Backer O, et al. Outcomes in Transcatheter Aortic Valve Replacement for Bicuspid Versus Tricuspid Aortic Valve Stenosis. J Am Coll Cardiol 2017;69:2579-89.

11. Puls M, Eiffert H, Hünlich M, R et al. Prosthetic valve endocarditis after transcatheter aortic valve implantation: the incidence in a single-centre cohort and reflections on clinical, echocardiographic and prognostic features. Eurointervention 2013;8:1407-18.

12. Habib G, Lancellotti P, Antunes MJ, et al. ESC Scientific Document Group. 2015 ESC Guidelines for the management of infective endocarditis: The Task Force for the Management of Infective Endocarditis of the European Society of Cardiology (ESC). Endorsed by: European Association for Cardio-Thoracic Surgery (EACTS), the European Association of Nuclear Medicine (EANM). Eur Heart J 2015;36:3075-128.

13. Mestres CA, Paré JC, Miró JM; Working Group on Infective Endocarditis of the Hospital Clínic de Barcelona. Organization and Functioning of a Multidisciplinary Team for the Diagnosis and Treatment of Infective Endocarditis: A 30-year Perspective (1985-2014). Rev Esp Cardiol (Engl Ed) $2015 ; 68: 363-8$.

14. Yanagawa B, Pettersson GB, Habib G, et al. Surgical Management of Infective Endocarditis Complicated by Embolic Stroke: Practical Recommendations for Clinicians. Circulation 2016;134:1280-92.

15. Lalani $\mathrm{T}$, Cabell $\mathrm{CH}$, Benjamin DK, et al; International Collaboration on Endocarditis-Prospective Cohort Study (ICE-PCS) Investigators. Analysis of the impact of early surgery on in-hospital mortality of native valve endocarditis: use of propensity score and instrumental variable methods to adjust for treatment-selection bias. Circulation 2010;121:1005-13.
16. Kang DH, Kim YJ, Kim SH, et al. Early surgery versus conventional treatment for infective endocarditis. N Engl J Med 2012;366:2466-73.

17. Kang DH, Lee S, Kim YJ, et al. Long-Term Results of Early Surgery versus Conventional Treatment for Infective Endocarditis Trial. Korean Circ J 2016;46:846-50.

18. Lalani $\mathrm{T}$, Chu $\mathrm{VH}$, Park LP, et al. International Collaboration on Endocarditis-Prospective Cohort Study Investigators. In-hospital and 1-year mortality in patients undergoing early surgery for prosthetic valve endocarditis. JAMA Intern Med 2013;173:1495-504.

19. Falk V, Baumgartner H, Bax JJ, et al. 2017 ESC/EACTS Guidelines for the management of valvular heart disease. Eur J Cardiothorac Surg 2017;52:616-64

20. Toyoda N, Itagaki S, Tannous H, Egorova NN, Chikwe J. Bioprosthetic Versus Mechanical Valve Replacement for Infective Endocarditis: Focus on Recurrence Rates. Ann Thorac Surg 2018;106:99-106.

21. Feringa $\mathrm{HH}, \mathrm{Bax} J \mathrm{~J}$, Klein $\mathrm{P}$, et al. Outcome after mitral valve repair for acute and healed infective endocarditis. Eur J Cardiothorac Surg 2006;29:367-73.

22. David TE, Gavra G, Feindel CM, Regesta T, Armstrong S, Maganti MD. Surgical treatment of active infective endocarditis: a continued challenge. often they Gillinov AM, Diaz R, Blackstone EH, Pettersson GB, Sabik JF, Lytle BW et al. Double valve endocarditis. Ann Thorac Surg 2001;71:18749.

23. Gillinov AM, Diaz R, Blackstone EH, Pettersson GB, Sabik JF, Lytle BW et al. Double valve endocarditis. Ann Thorac Surg 2001;71:1874-9.

24. Sheikh AM, Elhenawy AM, Maganti M, Armstrong S, David TE, Feindel CM. Outcomes of double valve surgery for active infective endocarditis. J Thorac Cardiovasc Surg 2009;138:69-75.

25. Goodwin AT, Ooi A, Kitcat J, Nashef SA. Outcomes in emergency redo cardiac surgery: cost, benefit and risk assessment. Interact Cardiovasc Thorac Surg 2003;2:227-30

26. Musci M, Weng Y, Hübler M, et al. Homograft aortic root replacement in native or prosthetic active infective endocarditis: twenty-year single-center experience. J Thorac Cardiovasc Surg 2010;139:665-73.

27. Elgharably H, Hakim AH, Unai S, et al. The incorporated aortomitral homograft for double-valve endocarditis: the 'hemi-Commando' procedure. Early and mid-term outcomes. Eur J Cardiothorac Surg 2018;53:1055-61.

28. Navia JL, Elgharably H, Hakim AH, et al. Long-term Outcomes of Surgery for Invasive Valvular Endocarditis Involving the Aortomitral Fibrosa. Ann Thorac Surg 2019;108:1314-23.

29. Quintana E, Mestres CA, Sandoval E, Ibáñez C, Van Hemelrijck M, Pomar JL. Infective aortic valve endocarditis with root abscess formation: a mitral sparing root-Commando operation. Ann Cardiothorac Surg 2019.

30. Aymami M, Revest M, Piau C, et al. Heart transplantation as salvage treatment of intractable infective endocarditis. Clin Microbiol Infect 2015;21:371.e1-4. 